\title{
Epicardial adipose tissue thickness and NGAL levels in women with polycystic ovary syndrome
}

\author{
Serap Baydur Sahin ${ }^{1,5^{*}}$, Medine Cumhur Cure ${ }^{2}$, Yavuz Ugurlu ${ }^{3}$, Elif Ergul ${ }^{3}$, Emine Uslu Gur ${ }^{4}$, Nese Alyildiz ${ }^{4}$ \\ and Mehmet Bostan ${ }^{3}$
}

\begin{abstract}
Background: Polycystic ovary syndrome (PCOS) is associated with an increased cardiovascular disease (CVD) risk and early atherosclerosis. Epicardial adipose tissue thickness (EATT) is clinically related to subclinical atherosclerosis. In the present study, considering the major role of neutrophil gelatinase-associated lipocalin (NGAL) which is an acute phase protein rapidly releasing upon inflammation and tissue injury, we aimed to evaluate NGAL levels and EATT in PCOS patients and assess their relationship with cardiometabolic factors.
\end{abstract}

Methods: 64 patients with PCOS and 50 age- and body mass index-matched healthy controls were included in the study. We evaluated anthropometric, hormonal and metabolic parameters. EATT was measured by echocardiography above the free wall of the right ventricle. Serum NGAL and high-sensitive C- reactive protein (hsCRP) levels were measured by ELISA.

Results: Mean EATT was 0,38 +/-0,16 mm in the PCOS group and 0,34+/-0,36 mm in the control group $(p=0,144)$. In the obese PCOS group $(n=44)$ EAT was thicker compared to the obese control group $(n=41)(p=0.026)$. Mean NGAL levels of the patients with PCOS were 101,98 +/-21,53 pg/ml, while mean NGAL levels were 107,40 +/-26,44 pg/ml in the control group $(p=0,228)$. We found a significant positive correlation between EATT and age, BMl, waist circumference, fasting insulin, HOMA-IR, triglyceride and hsCRP levels in PCOS group.

Conclusions: Thickness of the epicardial adipose tissue can be used to follow the risk of CVD development in obese PCOS cases. However serum NGAL levels do not differ in patients with PCOS and control group.

Keywords: Polycystic ovary syndrome, Epicardial adipose tissue thickness, NGAL

\section{Introduction}

Polycystic ovary syndrome (PCOS) is a common endocrine disorder that affects about $5-10 \%$ of women of reproductive age and characterized by hyperandrogenism, menstrual disturbance, chronic anovulation and infertility [1]. PCOS is frequently associated with multiple risk factors for coronary heart disease (CHD), including insulin resistance (IR), dyslipidemia, visceral obesity and hypertension [2,3]. It is reported to be associated with elevation of various markers of endothelial inflammation and abnormal endothelial function which plays a critical role in

\footnotetext{
* Correspondence: serapbaydur@gmail.com

${ }^{1}$ Department of Endocrinology and Metabolism Disease, Recep Tayyip Erdogan University Medical School, Rize, Turkey

${ }^{5}$ Department of Endocrinology and Metabolism Disease, Recep Tayyip Erdogan University Training and Research Hospital, 53020 Rize, Turkey Full list of author information is available at the end of the article
}

cardiovascular disease and in particular in the development and progression of atherosclerosis [4].

Neutrophil gelatinase-associated lipocalin (NGAL), also known as lipocalin-2 is an acute phase protein, that is rapidly released from neutrophils upon inflammation and tissue injury [5]. Additionally, it has been found to be expressed in several types of cells, including kidney tubular cells, adipocytes, macrophages, brain endothelial cells and hepatocytes [6,7]. NGAL has been implicated in some pathophysiological processes, including apoptosis, iron transport, inflammation, cell survival, tumorigenesis, and atherosclerosis [6,7]. Evidence suggests that NGAL plays an important role in systemic insulin sensitivity and glucose homeostasis [8,9]. In a recent study, NGAL was determined to reflect the degree of inflammatory process in coronary artery disease [10]. 
Epicardial adipose tissue is true visceral fat deposited around the heart, between the myocardium and visceral epicard [11]. It mediates atherosclerosis via expression of several bioactive molecules [12] and has been reported to reflect the intraabdominal visceral fat [13]. Epicardial adipose tissue thickness (EATT) has been demonstrated to correlate with the severity of coronary artery disease (CAD) and the extent of coronary artery atherosclerosis [14-16].

In this study, our aim was to measure NGAL levels and EATT in women with PCOS and compare them with those of age and body mass index (BMI)-matched controls and to evaluate their relationship with cardiometabolic factors.

\section{Materials and methods Study population}

This study included 66 patients with PCOS and age- body mass index (BMI) matched 50 healthy controls. Women were all of the same ethnicity. All the patients gave a written consent. This study was approved by the Institutional Ethical Committee of the institution in which this study was conducted. All the patients were nonsmokers.

The diagnosis of PCOS was made according to the Rotterdam European Society for Human reproduction and Embryology/American Society for Reproductive Medicine- sponsored PCOS Consensus Workshop Group [17]. The revised diagnostic criteria of PCOS is as follows, with at least two of the following being required: i) Oligo and/or anovulation that is menstrual disturbance, ii) Clinical and/or biochemical signs of hyperandrogenism, iii) Polycystic ovarian appearance on ultrasound examination. Clinical hirsutism was defined by a modified FerrimanGallwey score of more than eight.

Patients and healthy subjects who had smoking history, diabetes mellitus, thyroid disorders, hyperprolactinemia, non-classical congenital adrenal hyperplasia (NCAH), androgen-secreting tumours, Cushing syndrome (1 mg dexamethasone suppression test), infection diseases, hypertension, hepatic or renal dysfunction were excluded from the study. The other exclusion criteria were; drug use within 3 months such as oral contraceptive agents, antilipidemic and hypertensive drugs, and insulin-sensitizing drugs. For the exclusion of $\mathrm{NCAH}$, we measured $17 \mathrm{OH}-$ progesterone levels in the all patients. The patients who had basal $17 \mathrm{OH}$-progesterone levels $>4 \mathrm{ng} / \mathrm{ml}$ were diagnosed as NCAH. We performed ACTH stimulation test in patients who had $>2 \mathrm{ng} / \mathrm{ml}$ 17-OHP levels. The diagnosis of NCAH was considered in patients with the poststimulation 17-OHP level exceed $10 \mathrm{ng} / \mathrm{ml}$. These patients were not included in the study. We selected healthy volunteers from the subjects working at our hospital and students in our medical school and nursing school. Control group $(\mathrm{n}=50)$ consisted of healthy patients who had hirsutism score $<8$, regular menses, every 21-35 days and normal androgen levels None of the women in the control group had polycystic ovary in ultrasound.

\section{Clinical, biochemical and hormonal measurements}

Weight, height, waist circumference and systolic and diastolic blood pressure (BP) were measured. The BMI was calculated by dividing the body weight in kilograms by the square of the height in meters $\left(\mathrm{kg} / \mathrm{m}^{2}\right)$. Waist circumference was measured at the narrowest level between the costal margin and iliac crest. The BMI, waist circumference and hirsutism scores were assessed by a single investigator for all of the subjects.

Venous blood samples were obtained from all subjects following an $8-12 \mathrm{~h}$ overnight fast in the follicular phase of a spontaneous or progesterone induced menstrual cycle. The levels of fasting glucose, insulin, total cholesterol, triglyceride (TG), high-density lipoprotein cholesterol (HDL-cholesterol), low-density lipoprotein cholesterol (LDL-cholesterol) and high-sensitive C-reactive protein (hs-CRP) were measured. All the patients underwent a $75 \mathrm{gr}$ oral glucose tolerance test (OGTT). An insulin resistance score Homeostasis Model Assessment-Insulin resistance (HOMA-IR) was computed by the following formula [18]: HOMA-IR = fasting plasma glucose $(\mathrm{mmol} / \mathrm{L}) \times$ fasting serum insulin $(\mathrm{mU} / \mathrm{mL}) / 22.5$. The cut off value was taken 2.7 for HOMA-IR [19].

The hexokinase method was used to measure glucose levels, and photometric method (Abbott Architect c16000 otoanalyzer) was used to measure the total cholesterol, triglyceride (TG), high-density lipoprotein cholesterol (HDL-cholesterol), and low-density lipoprotein cholesterol (LDL-cholesterol) levels. Insulin was measured using CMIA (Chemiluminescent microparticle immunoassay) (Abbott, Architect system, USA). The concentration of high-sensitive C- reactive protein (hsCRP) was measured using immunotubidimetric method with Abbott Architect C16000 otoanalyzer (Abbott Diagnostic, USA). The cut off for hsCRP was taken $<0.5 \mathrm{mg} / \mathrm{dl}$.

Serum levels of insulin, follicle-stimulating hormone (FSH), luteinizing hormone (LH), prolactin, dehydroepiandrosterone sulfate (DHEAS), total testosterone, insulin and thyroid stimulating hormone (TSH) were measured using chemiluminescent microparticle enzyme immunoassay (CMIA) method with Abbott Architect i2000 (Abbott Diagnostic, USA). Serum 17 hydroxyprogesterone and free testosterone were measured by radioimmunoassay.

\section{Measurement of NGAL}

The serum levels of NGAL were measured using enzymelinked immunosorbent assay (ELISA) method. We used commercially available human NGAL ELISA kit (MyBiosource, USA). The procedure for the ELISA method was according to the instructions provided by the manufacturer. Absorbance was measured at a wavelength of $450 \mathrm{\eta m}$ using 
ELISA reader. The levels of NGAL are presented as $\mathrm{ng} / \mathrm{ml}$. The intra-assay and inter-assay coefficient of variation were $4.1 \%$ and $3.9 \%$, respectively. The limit of detection (LOD) for the NGAL assay was $<10 \mathrm{pg} / \mathrm{ml}$.

\section{Measurement of epicardial adipose tissue thickness}

All patients underwent 2D Doppler echocardiography with a Philips IE-33 system and S5-1 transducer $(1 \mathrm{e} 5 \mathrm{mHz}$, Philips, Bothell, WA, USA). The epicardial fat thickness (EFT) was identified as the echo-free space between the outer wall of the myocardium and the visceral layer of the pericardium, and its thickness was measured perpendicularly on the free wall of the right ventricle at end-systole in three cardiac cycles. Parasternal long- and short-axis views were used. The average value of three cardiac cycles from each echocardiographic view was considered [20,21]. All the measurements were done by the same cardiologist.

\section{Statistical analysis}

Data were analyzed using SPSS Software (Version 17, SPSS, Inc., Chicago, IL, USA). Results were expressed as mean \pm standard deviation. The Mann-Whitney $U$ test was used to compare the continuous variables and the Chi-square test was used to compare categorical variables. Spearman's rank correlation test was used for calculation of associations between variables. A p value of less than 0.05 was considered to be statistically significant.

\section{Results}

We enrolled 66 patients with PCOS (mean age; $23,29 \pm 5,05$ years, BMI; $\left.32,09 \pm 9,80 \mathrm{~kg} / \mathrm{m}^{2}\right)$ and 50 age and BMI matched healthy control group (mean age; $22,02 \pm 5,05$ years, BMI; $29,80 \pm 5,5 \mathrm{~kg} / \mathrm{m}^{2}$ ) in the current study. The clinical and biochemical results of the study population are shown in Table 1.

Fasting plasma glucose, hsCRP, triglyceride, total and LDL cholesterol levels were significantly higher in women with PCOS (Table 1).

Mean EATT was $0,38 \pm 0,16 \mathrm{~mm}$ in the PCOS group and $0,34 \pm 0,36 \mathrm{~mm}$ in the control group $(\mathrm{p}=0,144)$. Although EATT was higher in PCOS patients, the difference did not reach statistical significance. We found a significant correlation between EATT and age, BMI, waist circumference, fasting insulin, HOMA-IR, triglyceride and hsCRP in the patients with PCOS (Table 2). In the control group, EATT was also associated with.

Mean NGAL levels of the patients with PCOS were $101,98 \pm 21,53 \mathrm{pg} / \mathrm{ml}$, while mean NGAL levels were $107,40 \pm 26,44 \mathrm{pg} / \mathrm{ml}$ in the control group $(\mathrm{p}=0,228)$. Serum NGAL levels were not correlated with the metabolic parameters in both of the groups (Table 3).

The patients with PCOS were divided into 2 groups according to the HOMA-IR levels. The cut-off point was 2.7 [22]. 28 PCOS patients had an HOMA-IR greater
Table 1 The clinical, biochemical and hormonal results in women with polycystic ovary syndrome (PCOS) patients and healthy controls

\begin{tabular}{|c|c|c|c|}
\hline Parameter & $\begin{array}{l}\text { PCOS group } \\
(n=66)\end{array}$ & $\begin{array}{l}\text { Control group } \\
\qquad(n=50)\end{array}$ & $p$ \\
\hline Age (years) & $23.29 \pm 5.05$ & $22.02 \pm 5.05$ & 0.183 \\
\hline BMI (kg/m2) & $32.09 \pm 9.80$ & $29.80 \pm 5.5$ & 0.148 \\
\hline Waist circumference (cm) & $8.67 \pm 19.37$ & $92.28 \pm 13.30$ & 0.048 \\
\hline Systolic blood pressure (mmHg) & $126.77 \pm 14.15$ & $113.22 \pm 9.99$ & 0.0001 \\
\hline Diastolic blood pressure $(\mathrm{mmHg})$ & $76.18 \pm 11.37$ & $68.20 \pm 6.74$ & 0.0001 \\
\hline Fasting insulin ( $\mu \mathrm{l} \mathrm{U} / \mathrm{ml})$ & $11.16 \pm 6.58$ & $9.47 \pm 6.06$ & 0.160 \\
\hline Fasting glucose (mg/dl) & $97.30 \pm 13.97$ & $89.10 \pm 10.56$ & 0.001 \\
\hline HOMA-IR & $2.71 \pm 1.7$ & $2.16 \pm 1.5$ & 0.072 \\
\hline Total cholesterol (mg/dl) & $194.81 \pm 39.55$ & $173.28 \pm 29.99$ & 0,002 \\
\hline Triglyceride (mg/dl) & $115.91 \pm 58.18$ & $93.30 \pm 39.30$ & 0.020 \\
\hline $\mathrm{HDL}-\mathrm{C}(\mathrm{mg} / \mathrm{dl})$ & $49.61 \pm 11.09$ & $47.08 \pm 10.30$ & 0.213 \\
\hline LDL-C (mg/dl) & $122.83 \pm 34.43$ & $107.0 \pm 24.64$ & 0.007 \\
\hline hsCRP (mg/dl) & $0.54 \pm 0.95$ & $0.19 \pm 0.31$ & 0.015 \\
\hline TSH $(\mu \mathrm{lU} / \mathrm{ml})$ & $2.11 \pm 1.47$ & $2.58 \pm 3.24$ & 0.295 \\
\hline Prolactin (ng/ml) & $18.3 \pm 15.4$ & $17.4 \pm 6.9$ & 0.590 \\
\hline Total testosterone (ng/ml) & $0.85 \pm 0.3$ & $0.56 \pm 0.1$ & 0.0001 \\
\hline FSH (mlU/ml) & $4.5 \pm 1.2$ & $4.5 \pm 0.7$ & 0.950 \\
\hline LH (mlU/ml) & $5.7 \pm 2.7$ & $3.5 \pm 0.5$ & 0.0001 \\
\hline Estradiol (pg/ml) & $43.9 \pm 33.3$ & $41.02 \pm 3.1$ & 0.536 \\
\hline DHEAS ( $\mu \mathrm{g} / \mathrm{dl})$ & $266.3 \pm 123$ & $203.85 \pm 40.3$ & 0.0001 \\
\hline Free testosterone $(\mathrm{pg} / \mathrm{ml})$ & $3,3 \pm 2.3$ & $2.3 \pm 0.4$ & 0.008 \\
\hline EATT (mm) & $0.38 \pm 0.16$ & $0.34 \pm 0.36$ & 0.144 \\
\hline NGAL (pg/ml) & $101.98 \pm 21.53$ & $107.40 \pm 26.44$ & 0.228 \\
\hline
\end{tabular}

Values are expressed as means \pm SD. BMI body mass index, HOMA-IR homeostasis model assessment insulin resistance index, $\mathrm{HDL}-\mathrm{C}$ high density lipoprotein cholesterol, LDL-C low density lipoprotein cholesterol, hs-CRP high-sensitive $\mathrm{C}$ - reactive protein, $\mathrm{TSH}$ thyroid stimulating hormone, $\mathrm{FSH}$ follicle-stimulating hormone, LH luteinizing hormone, DHEAS dehydroepiandrosterone sulfat, EATT epicardial adipose tissue thickness, NGAL neutrophil gelatinase-associated lipocalin.

than 2.7, while 38 patients had an HOMA-IR lower than 2.7. EATT and hsCRP levels were higher in the insulin resistant group, however NGAL levels were similar in both of the groups (Table 4). The insulin resistant control group $(\mathrm{n}=14)$ had also higher hsCRP levels, but EATT was similar.

When we evaluate the PCOS patients according to the hsCRP values, 16 patients had hsCRP values higher than $0.5 \mathrm{mg} / \mathrm{L}$ and EATT was found to be higher in this subgroup $(\mathrm{p}=0.007)$. In the control group, 4 patients had hsCRP levels $\geq 0.5 \mathrm{mg} / \mathrm{L}$ and EATT was similar in the PCOS and control group. NGAL levels did not differ in both of the groups according to hsCRP levels.

When we divide the PCOS patients according to BMI, 21 patients had $\mathrm{BMI}<25 \mathrm{~kg} / \mathrm{m}^{2}$. We compared the lean and obese patients with PCOS and found that NGAL and 
Table 2 The correlation between epicardial adipose tissue thickness and metabolic parameters in patients with PCOS and control group

\begin{tabular}{|c|c|c|c|c|}
\hline \multirow[t]{2}{*}{ Parameter } & \multicolumn{2}{|c|}{ PCOS group $(n=66)$} & \multicolumn{2}{|c|}{ Control group $(n=50)$} \\
\hline & $r$ & $p$ value & $r$ & $p$ value \\
\hline Age & 0,248 & $0,045^{*}$ & 0,231 & 0,106 \\
\hline BMI & 0,641 & $0,0001^{* *}$ & 0,314 & $0,027^{*}$ \\
\hline WC & 0,563 & $0,0001^{* *}$ & 0,258 & 0,070 \\
\hline FG score & 0,061 & 0,626 & & \\
\hline Fasting glucose & 0,072 & 0,565 & 0,137 & 0,342 \\
\hline Fasting insulin & 0,472 & $0,0001^{* *}$ & 0,088 & 0,545 \\
\hline HOMA-IR & 0,641 & $0,0001^{* *}$ & 0,300 & $0,034^{*}$ \\
\hline Total cholesterol & 0,216 & 0,081 & 0,299 & $0,035^{*}$ \\
\hline Triglyceride & 0,363 & $0,003^{* *}$ & 0,382 & $0,006^{* *}$ \\
\hline LDL cholesterol & 0,213 & 0,085 & 0,274 & 0,054 \\
\hline HDL cholesterol & $-0,200$ & 0,108 & $-0,243$ & 0,093 \\
\hline hsCRP & 0,324 & $0,008^{* *}$ & 0,096 & 0,507 \\
\hline NGAL & 0,086 & 0,494 & $-0,051$ & 0,727 \\
\hline TSH & $-0,110$ & 0,377 & 0,010 & 0,946 \\
\hline Estradiol & 0,026 & 0,834 & 0,029 & 0,846 \\
\hline LH/FSH & $-0,367$ & 0,068 & & \\
\hline DHEAS & $-0,133$ & 0,286 & $-0,011$ & 0,939 \\
\hline Total testosterone & 0,185 & 0,137 & 0,078 & 0,596 \\
\hline
\end{tabular}

$\mathrm{r}$ indicates Spearman's rho correlation coefficient; ${ }^{*}$ Correlation is significant at the 0.05 level. ${ }^{*}$ Correlation is significant at the 0.01 level. a log transformed. BMI body mass index, HOMA-IR homeostasis model assessment insulin resistance index, HDL-C high density lipoprotein cholesterol, LDL-C low density lipoprotein cholesterol, hs-CRP high-sensitive C- reactive protein, NGAL neutrophil gelatinase-associated lipocalin, TSH thyroid stimulating hormone, FSH follicle-stimulating hormone, LH luteinizing hormone, DHEAS dehydroepiandrosterone sulfat.

hsCRP levels were similar in both of the groups. However HOMA-IR and EATT was higher in the obese patients $(\mathrm{p}=0.001, \mathrm{p}=0.007)$. In the control group, 41 patients had BMI $>25 \mathrm{~kg} / \mathrm{m}^{2}$ and EATT, serum NGAL and hsCRP levels did not differ in the obese and healthy subjects $(\mathrm{p}=0.902, \mathrm{p}=0.057, \mathrm{p}=0.342)$. When we compared the obese PCOS $(\mathrm{n}=44)$ and obese control group $(\mathrm{n}=41)$, EAT was thicker in the PCOS group $(\mathrm{p}=$ 0.026), however NGAL levels did not differ (Table 5).

\section{Discussion}

Our study shows that epicardial adipose tissue thickness is higher in obese PCOS patients compared with obese control subjects, however NGAL levels are similar in both of the groups. We demonstrated that EATT was positively correlated with age, BMI, waist circumference, fasting insulin, HOMA-IR, triglyceride and hsCRP levels. Therefore, EATT can be used to follow the risk of CVD development in obese PCOS cases.

Women with PCOS have multiple risk factors for cardiovascular disease (CVD) and is considered as a major
Table 3 The correlation between NGAL levels and metabolic parameters in patients with PCOS

\begin{tabular}{lcc}
\hline Parameter & $\mathbf{r}$ & $\mathbf{p}$ value \\
\hline Age & 0,042 & 0,738 \\
BMI & 0,149 & 0,235 \\
WC & 0,148 & 0,239 \\
FG score & 0,051 & 0,684 \\
Fasting glucose & 0,088 & 0,487 \\
Fasting insulin & $-0,109$ & 0,880 \\
HOMA-IR & 0,031 & 0,808 \\
Total cholesterol & 0,013 & 0,917 \\
Triglyceride & 0,037 & 0,772 \\
LDL cholesterol & 0,019 & 0,882 \\
HDL cholesterol & $-0,081$ & 0,523 \\
hsCRP & 0,064 & 0,612 \\
EATT & 0,086 & 0,494 \\
TSH & 0,176 & 0,162 \\
Estradiol & $-0,001$ & 0,993 \\
LH/FSH & $-0,089$ & 0,702 \\
DHEAS & 0,216 & 0,085 \\
Total testosteron & $-0,043$ & 0,733 \\
\hline r indicates Spearman's rho correlation coefficient. BMI body mass index, \\
HOMA-IR homeostasis model assessment insulin resistance index, HDL-C high \\
density lipoprotein cholesterol, LDL-C low density lipoprotein cholesterol, \\
hs-CRP high-sensitive C- reactive protein, EAT epicardial adipose tissue \\
thickness, TSH thyroid stimulating hormone, FSH follicle-stimulating hormone, \\
LH luteinizing hormone, DHEAS dehydroepiandrosterone sulfat. & \\
& & \\
\hline
\end{tabular}

long-term health risk. The risk factors for premature atherosclerosis and CVD, such as increased central adiposity, elevated blood pressure, higher total and LDL cholesterol levels, higher triglyceride levels, and higher plasma glucose levels were more prevalent in our PCOS population, compared with matched-for-age and BMI control women, as have been shown in previous studies $[23,24]$. These metabolic risk factors may predict the development of atherosclerosis in women with PCOS.

Although an increase in mortality due to coronary artery disease in patients with PCOS was not shown in the long term follow up [2], there is strong evidence that women with PCOS are at increased risk of metabolic cardiovascular syndrome [25]. In a study, coronary artery calcification was evaluated in younger women with PCOS $30-45$ years as a measure of subclinical atherosclerotic disease burden, and it was found to be more prevalent in PCOS women [3]. In the other study, postmenopausal women noted 2.5 higher odds of obstructive coronary artery disease for those with clinical features of PCOS, even when controlling for metabolic and common cardiac risk factors [26].

Highly sensitive C-reactive protein (hsCRP) levels are good predictors of subclinical atherosclerosis and vascular 
Table 4 The comparison of PCOS and control group according to HOMA-IR levels

\begin{tabular}{|c|c|c|c|c|c|c|}
\hline Variable & $\begin{array}{l}\text { HOMA-IR } \geq 2.7 \\
\text { PCOS group } \\
(n=28)\end{array}$ & $\begin{array}{c}\text { HOMA-IR }<2.7 \\
\text { PCOS group } \\
(n=38)\end{array}$ & $p$ & $\begin{array}{l}\text { HOMA-IR } \geq 2.7 \\
\text { Control group } \\
\qquad(n=14)\end{array}$ & $\begin{array}{c}\text { HOMA-IR }<2.7 \\
\text { Control group } \\
\quad(n=36)\end{array}$ & $p$ \\
\hline EATT (mm) & $0.44 \pm 1.1$ & $0.34 \pm 1.4$ & 0.019 & $0.35 \pm 0.16$ & $0.33 \pm 0.12$ & 0.592 \\
\hline NGAL (pg/ml) & $100.4 \pm 23.2$ & $103.1 \pm 20.3$ & 0.610 & $113.5 \pm 21.4$ & $105 \pm 28.1$ & 0.310 \\
\hline hsCRP (mg/dl) & $0.89 \pm 1.1$ & $0.27 \pm 0.6$ & 0.009 & $0.38 \pm 0.5$ & $0.11 \pm 0.14$ & 0.004 \\
\hline BMI (kg/m2) & $37.5 \pm 10.3$ & $28.1 \pm 7.3$ & 0.0001 & $34.06 \pm 5.5$ & $28.1 \pm 4.6$ & 0.0001 \\
\hline
\end{tabular}

Values are expressed as means \pm SD.

events [22]. Many studies have also shown high hsCRP levels in women with PCOS [22,27] in contrast to others that say it is related only to obesity [28]. In our study, serum hsCRP levels were significantly higher in women with PCOS than the controls and it was correlated with BMI, EATT and decreased insulin sensitivity. Kim et al. have reported higher hsCRP levels even in lean PCOS women [22]. In our study, hsCRP levels did not differ in lean PCOS and lean control subjects.

NGAL is highly expressed in adipocytes, that its expression is regulated by obesity, and that it induces insulin resistance [8]. Studies investigating the relationship between NGAL levels and PCOS found confusing results. Cakal E et al. demonstrated that NGAL levels were higher in PCOS patients and was related with insulin resistance [29]. However, in the other study, NGAL levels were found to be lower in PCOS patients [30]. In our study, NGAL levels were similar in PCOS patients and healthy subjects. NGAL measurements also did not differ in groups based on the HOMA-IR and hsCRP levels and BMI.

In the recent studies it was found that serum levels of NGAL were significantly elevated in patients with angiographically confirmed CAD compared to those with normal arteries or controls [10,31,32]. The authors explain that these diffrences can result because of the expression of NGAL from vascular cells during atherogenesis. In our study, NGAL levels did not correlate with the cardiometabolic risk factors.

Epicardial adipose tissue thickness is clinically related to abdominal visceral adiposity which has significant impact on cardiovascular diasease risk [13]. In recent

Table 5 The comparison of obese PCOS and control subjects

\begin{tabular}{lccc}
\hline & $\begin{array}{c}\text { Obese PCOS group } \\
(\mathbf{n}=\mathbf{4 4})\end{array}$ & $\begin{array}{c}\text { Obese control group } \\
(\mathbf{n}=\mathbf{4 1})\end{array}$ & $\boldsymbol{p}$ \\
\hline EATT $(\mathrm{mm})$ & $0.42 \pm 0.1$ & $0.34 \pm 0.1$ & 0.026 \\
NGAL $(\mathrm{pg} / \mathrm{ml})$ & $104.2 \pm 22.9$ & $110.7 \pm 27.1$ & 0.235 \\
HOMA-IR & $3.1 \pm 1.7$ & $2.4 \pm 1.5$ & 0.045 \\
hsCRP $(\mathrm{mg} / \mathrm{dl})$ & $0.62 \pm 0.9$ & $0.21 \pm 0.33$ & 0.012 \\
\hline
\end{tabular}

Values are expressed as means \pm SD. studies, EATT was found to be associated with coronary heart diseases [14-16].

There are only few studies evaluating the EATT in PCOS patients and in both of the studies EAT was thicker in the PCOS patients [33,34]. In our study, EAT was thicker in the PCOS group, but the difference was not significant. EATT was associated with the cardiometabolic risk factors, such as HOMA-IR and triglyceride in PCOS and control group. EATT was also associated with hsCRP in the PCOS patients, while it was not in the control group. When we compared the PCOS and control group according to HOMA-IR, hsCRP levels and BMI, we showed that EAT was thicker in the PCOS group who were obese and had higher HOMA-IR and hsCRP levels. However we did not demonstrate such a relationship in the control group. Additionally, in obese PCOS patients EATT was higher compared to obese healthy subjects. All obese subjects, whether PCOS or controls had similar EATT compared to the lean subjects. Therefore, we suggest that obesity does not solely influence EATT.

In conclusion, measurement of epicardial adipose tissue thickness is a non-invasive tool and easy to perform and appears to be a good method to follow the risk of cardiovascular disease development in obese and insulin resistant PCOS patients.

\section{Competing interests}

The authors declare that they have no competing interests.

\section{Authors' contributions}

SBS, MCC and MB conceived the study and participated in its design. YU and EE performed the ultrasound examinations. SBS, EUG, NA and MB participated in the analysis and interpretation of data. SBS, NA and EUG collected the data. SBS, MCC, YU, EE, EUG, NA and MB wrote the paper. All authors read and approved the final manuscript.

\section{Author details}

${ }^{1}$ Department of Endocrinology and Metabolism Disease, Recep Tayyip Erdogan University Medical School, Rize, Turkey. ${ }^{2}$ Department of Biochemistry, Recep Tayyip Erdogan University Medical School, Rize, Turkey. ${ }^{3}$ Department of Cardiology, Recep Tayyip Erdogan University Medical School, Rize, Turkey. ${ }^{4}$ Department of Internal Medicine, Recep Tayyip Erdogan University Medical School, Rize, Turkey. ${ }^{5}$ Department of Endocrinology and Metabolism Disease, Recep Tayyip Erdogan University Training and Research Hospital, 53020 Rize, Turkey. 
Received: 30 October 2013 Accepted: 11 February 2014

Published: 16 February 2014

\section{References}

1. Azziz R, Woods KS, Reyna R, Key TJ, Knochenhauer ES, Yildiz BO: The prevalence and features of the polycystic ovary syndrome in an unselected population. J Clin Endocrinol Metab 2004, 89(6):2745-2749.

2. Wild S, Pierpoint T, McKeigue P, Jacobs H: Cardiovascular disease in women with polycystic ovary syndrome at long-term follow-up: a retrospective cohort study. Clin Endocrinol (Oxf) 2000, 52(5):595-600.

3. Christian RC, Dumesic DA, Behrenbeck T, Oberg AL, Sheedy PF 2nd, Fitzpatrick LA: Prevalence and predictors of coronary artery calcification in women with polycystic ovary syndrome. J Clin Endocrinol Metab 2003, 88(6):2562-2568

4. Ross R: Atherosclerosis-an inflammatory disease. N Engl J Med 1999, 340(2):115-126.

5. Schmidt-Ott KM, Mori K, Li JY, Kalandadze A, Cohen DJ, Devarajan P, et al: Dual action of neutrophil gelatinase-associated lipocalin. J Am SoC Nephrol 2007, 18(2):407-413.

6. Chen X, Jia X, Qiao J, Guan Y, Kang J: Adipokines in reproductive function: a link between obesity and polycystic ovary syndrome. Mol Endocrinol 2013, 50(2):R21-R37.

7. Zhang J, Wu Y, Zhang Y, Leroith D, Bernlohr DA, Chen X: The role of lipocalin 2 in the regulation of inflammation in adipocytes and macrophages. Mol Endocrinol 2008, 22(6):1416-1426.

8. Yan QW, Yang Q, Mody N, Graham TE, Hsu CH, Xu Z, et al: The adipokine lipocalin 2 is regulated by obesity and promotes insulin resistance. Diabetes 2007, 56(10):2533-2540.

9. Wang Y, Lam KS, Kraegen EW, Sweeney G, Zhang J, Tso AW, et al: Lipocalin-2 is an inflammatory marker closely associated with obesity, insulin resistance, and hyperglycemia in humans. Clin Chem 2007, 53(1):34-41.

10. Kafkas N, Demponeras C, Zoubouloglou F, Spanou L, Babalis D, Makris K: Serum levels of gelatinase associated lipocalin as indicator of the inflammatory status in coronary artery disease. Int J Inflam 2012, 2012:189797.

11. lacobellis G, Corradi D, Sharma AM: Epicardial adipose tissue: anatomic, biomolecular and clinical relationships with the heart. Nat Clin Pract Cardiovasc Med 2005, 2(10):536-543.

12. Baker AR, Silva NF, Quinn DW, Harte AL, Pagano D, Bonser RS, et al: Human epicardial adipose tissue expresses a pathogenic profile of adipocytokines in patients with cardiovascular disease. Cardiovasc Diabetol 2006, 5:1.

13. lacobellis G, Assael F, Ribaudo MC, Zappaterreno A, Alessi G, Di Mario U, et al: Epicardial fat from echocardiography: a new method for visceral adipose tissue prediction. Obes Res 2003, 11(2):304-310.

14. Ahn SG, Lim HS, Joe DY, Kang SJ, Choi BJ, Choi SY, et al: Relationship of epicardial adipose tissue by echocardiography to coronary artery disease. Heart 2008, 94(3):e7.

15. Eroglu S, Sade LE, Yildirir A, Bal U, Ozbicer S, Ozgul AS, et al: Epicardial adipose tissue thickness by echocardiography is a marker for the presence and severity of coronary artery disease. Nutr Metab Cardiovasc Dis 2009, 19(3):211-217.

16. Djaberi R, Schuijf JD, van Werkhoven JM, Nucifora G, Jukema JW, Bax JJ: Relation of epicardial adipose tissue to coronary atherosclerosis. Am J Cardiol 2008, 102(12):1602-1607.

17. Rotterdam ESHRE/ASRM-Sponsored PCOS Consensus Workshop Group: Revised 2003 consensus on diagnostic criteria and long-term health risks related to polycystic ovary syndrome. Fertil Steril 2004, 81:19-25.

18. Matthews DR, Hosker JP, Rudenski AS, Naylor BA, Treacher DF, Turner RC Homeostasis model assessment: insulin resistance and beta-cell function from fasting plasma glucose and insulin concentrations in man. Diabetologia 1985, 28:412-419.

19. Gokcel A, Ozsahin AK, Sezgin N, et al: High prevalence of diabetes in Adana, a southern province of Turkey. Diabetes Care 2003, 26:3031-3034.

20. lacobellis G, Willens HJ, Barbaro G, Sharma AM: Threshold values of high-risk echocardiographic epicardial Fat thickness. Obesity (Silver Spring) 2008, 16(4):887-892.

21. Mariani S, Fiore D, Barbaro G, Basciani S, Saponara M, D'Arcangelo E, et al: Association of epicardial fat thickness with the severity of obstructive sleep apnea in obese patients. Int J Cardiol 2013, 167(5):2244-2249.
22. Kim JW, Han JE, Kim YS, Won HJ, Yoon TK, Lee WS: High sensitivity C-reactive protein and its relationship with impaired glucose regulation in lean patients with polycystic ovary syndrome. Gynecol Endocrinol 2012, 28(4):259-263.

23. Talbott E, Guzick D, Clerici A, Berga S, Detre K, Weimer K, et al: Coronary heart disease risk factors in women with polycystic ovary syndrome. Arterioscler Thromb Vasc Biol 1995, 15(7):821-826.

24. Graf M, Richards C, Brown V, Meissner L, Dunaif A: The independent effects of hyperandrogenaemia, hyperinsulinaemia and obesity on lipid and lipoprotein profiles in women. Clin Endocrinol (Oxf) 1990, 33(1):119-131.

25. Talbott EO, Zborowski JV, Rager JR, Boudreaux MY, Edmundowicz DA Guzick DS: Evidence for an association between metabolic cardiovascular syndrome and coronary and aortic calcification among women with polycystic ovary syndrome. J Clin Endocrinol Metab 2004, 89:5454-5461.

26. Shaw L, Bairey Merz CN, Azziz R, Stanczyk FZ, Sopko G, Braunstein GD, et al Postmenopausal women with a history of irregular menses and elevated androgen measurements at high risk for worsening cardiovascular event-free survival: results from the national institutes of health-national heart, lung, and blood institute sponsored Women's ischemia syndrome evaluatio. J Clin Endocrinol Metab 2008, 93(4):1276-1284.

27. Talbott EO, Zborowski JV, Boudreaux MY, McHugh-Pemu KP, Sutton-Tyrrell K, Guzick DS: The relationship between C-reactive protein and carotid intima-media wall thickness in middle-aged women with polycystic ovary syndrome. J Clin Endocrinol Metab 2004, 89(12):6061-6067.

28. Ketel IJ, Stehouwer CD, Henry RM, Serné EH, Hompes P, Homburg R, et al: Greater arterial stiffness in polycystic ovary syndrome (PCOS) is an obesity-but not a PCOS-associated phenomenon. J Clin Endocrinol Metab 2010, 95(10):4566-4575.

29. Cakal E, Ozkaya M, Engin-Ustun Y, Ustun Y: Serum lipocalin-2 as an insulin resistance marker in patients with polycystic ovary syndrome. J Endocrinol Invest 2011, 34(2):97-100.

30. Diamanti-Kandarakis E, Livadas S, Kandarakis SA, Margeli A, Papassotiriou I: Serum concentrations of atherogenic proteins neutrophil gelatinaseassociated lipocalin and its complex with matrix metalloproteinase-9 are significantly lower in women with polycystic ovary syndrome: hint of a protective mechanism? Eur J Endocrinol 2008, 158(4):525-531.

31. Choi KM, Lee JS, Kim EJ, Baik SH, Seo HS, Choi DS, et al: Implication of lipocalin-2 and visfatin levels in patients with coronary heart disease. Eur J Endocrinol 2008, 158(2):203-207.

32. Zografos T, Haliassos A, Korovesis $\mathrm{S}$, Giazitzoglou E, Voridis E, Katritsis D: Association of neutrophil gelatinase-associated lipocalin with the severity of coronary artery disease. Am J Cardiol 2009, 104(7):917-920.

33. Aydogdu A, Uckaya G, Tasci I, Baysan O, Tapan S, Bugan B, et al: The relationship of epicardial adipose tissue thickness to clinical and biochemical features in women with polycystic ovary syndrome. Endocr $\lrcorner$ 2012, 59(6):509-516.

34. Cakir E, Doğan M, Topaloglu O, Ozbek M, Cakal E, Vural MG, et al: Subclinical atherosclerosis and hyperandrogenemia are independent risk factors for increased epicardial fat thickness in patients with PCOS and idiopathic hirsutism. Atherosclerosis 2013, 226(1):291-295.

doi:10.1186/1757-2215-7-24

Cite this article as: Sahin et al.: Epicardial adipose tissue thickness and NGAL levels in women with polycystic ovary syndrome. Journal of Ovarian Research 2014 7:24.

\section{Submit your next manuscript to BioMed Central and take full advantage of:}

- Convenient online submission

- Thorough peer review

- No space constraints or color figure charges

- Immediate publication on acceptance

- Inclusion in PubMed, CAS, Scopus and Google Scholar

- Research which is freely available for redistribution 